\title{
Incidental durotomy in spine surgery: first aid in ten steps
}

\author{
Luca Papavero • Nils Engler · Ralph Kothe
}

Received: 19 October 2014/Revised: 18 January 2015/Accepted: 23 February 2015/Published online: 4 March 2015

(C) The Author(s) 2015. This article is published with open access at Springerlink.com

\begin{abstract}
Introduction Incidental durotomy (ID) is the most common complication of spine surgery. Revision procedures, ossification of the yellow ligament, or synovial cysts are well-known risk factors. The size, shape, and severity of ID are unpredictable, ranging from a pinpoint hole to a several centimeters large dural laceration with transected fibers following the slippage of a cutting burr. Furthermore, the occurrence of ID is always unexpected. Intra-operative management is often based on a steep learning curve rather than a structured scheme.

Purpose To provide an intra-operative ten-step closure technique (10ST) for IDs of varying severity.

Methods A database of 4020 consecutive surgeries for lumbar degenerative disease over the past 4 years was searched for ID. The records of 176 patients were analyzed. Two dural repair techniques were compared: the "individual" technique (InT) and the 10ST.

Results The overall prevalence of ID was $4.4 \%$. The prevalence was lowest in virgin micro-discectomies $(1.7 \%)$ and ranged from $3.6 \%$ in decompression for spinal canal stenosis up to $14.5 \%$ in revision procedures. All surgeries were performed with the aid of a microscope. Among 107 primary surgeries, the InT achieved a singlestage closure of the ID in 96 procedures (89.7\%). Among 20 virgin surgeries, the $10 \mathrm{ST}$ was successful in all cases $(P=0.21)$. Among 42 re-do procedures following failed
\end{abstract}

L. Papavero $(\bowtie) \cdot$ N. Engler $\cdot$ R. Kothe

Clinic for Spine Surgery, Schoen Klinik Hamburg Eilbek,

Dehnhaide 120, 22081 Hamburg, Germany

e-mail: lpapavero@schoen-kliniken.de attempts to stop cerebrospinal fluid (CSF) leakage, the InT achieved single-stage closure in 36 procedures $(85.7 \%)$. The 10ST was successful in all 26 cases $(P=0.03)$. The follow-up was 1 year.

Conclusions The 10ST should be considered for successful single-stage closure in primary repair of ID.

Keywords Incidental durotomy - Complications in spine surgery - Dural tear - Cerebrospinal fluid leak - Surgical education

\section{Introduction}

Data concerning the relationship between the years of attending experience and the rate of ID are conflicting. Some authors have reported an inverse relationship [1, 2], whereas others have reported "a baseline rate of durotomies unrelated to years of training" [3]. One explanation for the discrepancy is that situations favoring ID are better anticipated and prevented with increasing surgical experience. On the other hand, experienced surgeons have to deal with higher risk procedures, such as revision surgery or complex spine surgery $[4,5]$.

The prevalence of ID in spine surgery varies widely. In virgin lumbar surgery, the prevalence of ID ranges from 3 to $5 \%$; whereas in lumbar revision procedures, it ranges from 7 to $17 \%[3,6,7,8]$. The variation may be due to the difference between microsurgical procedures and conventional open techniques. However, ID is thought to be the most common complication of spine surgery, with the lumbar area as the most frequent location [3, 7]. Primary repair of ID is the first choice treatment and, if successful, the long-term clinical outcome is excellent $[3,9-11]$. 
Specific features of ID are:

1. Unpredictable size, shape, and severity-the injury may range from a pinpoint hole of the thecal sac to a several centimeter large dural laceration with transected caudal fibers following the slippage of a cutting burr (Fig. 1).

2. Unexpected occurrence, especially at the beginning of a surgeon's career-intra-operative management relies more on an emotionally intense and steep learning curve than the step-by-step implementation of a treatment plan.

Traditionally, ID closure has not been a structural part of the teaching program for spinal trainees at our institution. In 2010, a ten-step guideline with the acronym "Bird Dove, MD" was introduced as a tutorial in the residency program.

The goal of the present study was to compare the efficacy of the 10ST with the InT of the "old days". These latter techniques varied slightly amongst the surgeons but they had the following features in common:

- No intradural patching of the ID to stop the pulsatile force of CSF

- Direct closure of the dural tear by suturing or indirect closure by patching

- Epidural fibrin glue and/or free muscle or fat tissue
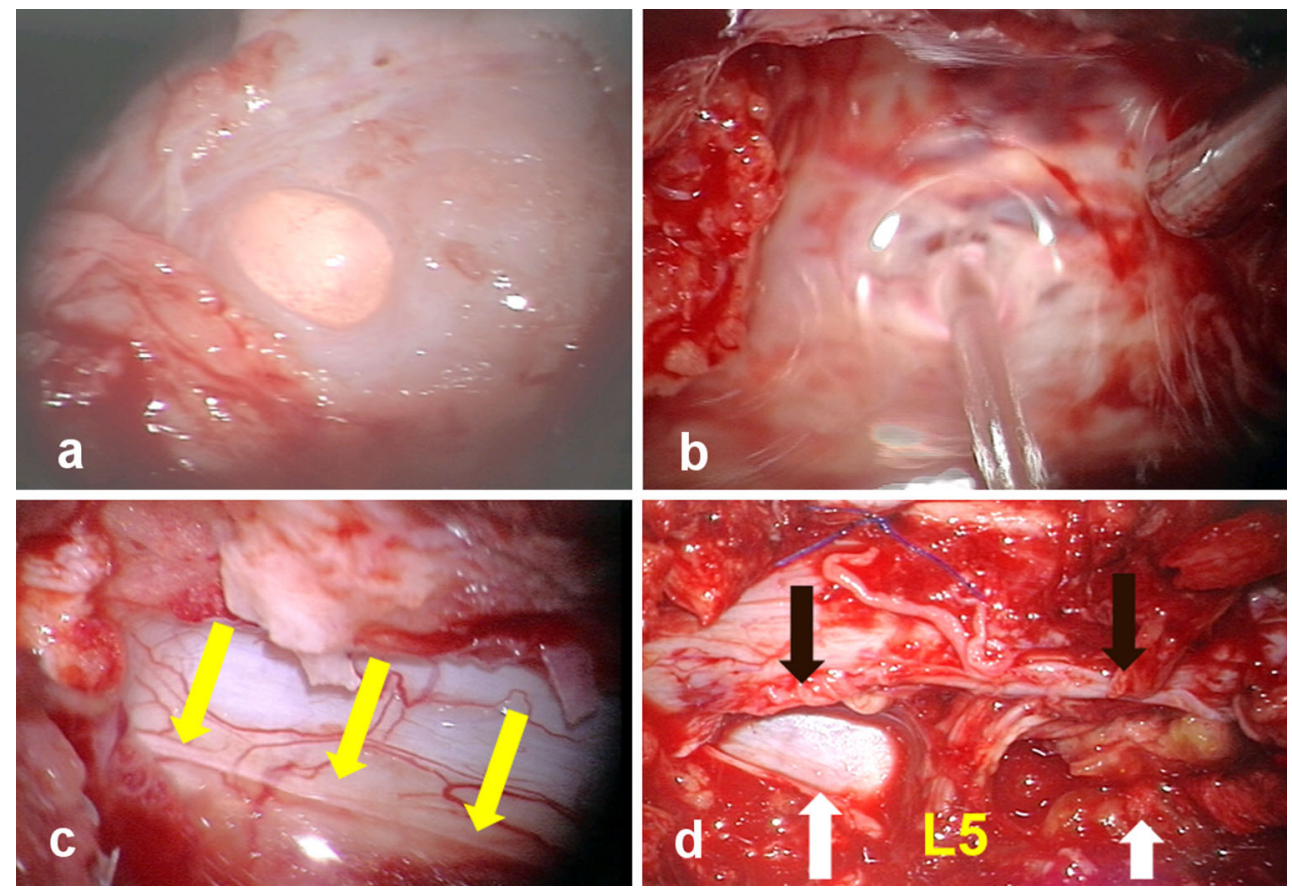

Fig. 1 a ID following injury by a diamond dust-coated burr. The arachnoid is still preserved. b Circular defect by offense with a cutting burr. The arachnoid was also severed. c Slit (yellow arrows)

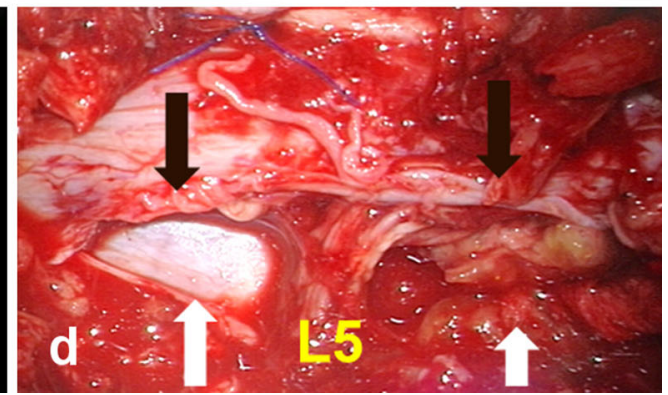
arrows)-lateral (white arrows) laceration with naked L5 root
- Fascial closure but no deep anchoring of ae pedicled paraspinal muscle flap

- CSF drainage cephalad to the ID when the Valsalva maneuver had shown some CSF spillage not controlled by other means.

\section{Methods}

Patients

Data from 4020 surgeries performed for lumbar degenerative diseases between February 2007 and May 2013 were analyzed. A total of 176 patients (100 females, 76 males, mean age 58 years) enclosing 13 patients with persistent lumbar CSF fistula referred to us from other Spine Centers were treated for ID in 195 surgical procedures. Several patients needed a second and few even a third look to get a successful closure of the ID. Six board-certified orthopedic surgeons and two board-certified neurosurgeons performed the surgeries. The intraspinal part of the surgery was performed routinely with the aid of a microscope. In 149 procedures, the surgeons repaired the ID with the InT with which they felt most confident. The 10ST was applied in 46 procedures. The patients were examined clinically 1 year after the last surgery.

following a bite with a punch and pulling it off. d Large dorso (black 
Statistical analysis

Significance was evaluated using the Student-Fisher $t$ test and established as $P \leq 0.05$.

Ten-step closure technique

The intra-operative repair of an ID started by positioning the patient so that the CSF leak was the highest point of the surgical situs (Fig. 2).

A microscope and selected instruments (Fig. 3) were useful in our hands. To localize the CSF leak whenever the dural tear was not obvious, fluorescein was injected intrathecally a spinal level above the surgical situs. A collagen fleece with human thrombin and fibrinogen on one side (Tachosil ${ }^{\circledR}$, Takeda $\mathrm{GmbH}$, Germany) provided a tight dural closure up to a pressure of $62 \mathrm{~cm} \mathrm{H}_{2} \mathrm{O}$.

The 10ST, summarized by the acronym "Bird Dove, MD", was applied as follows

1. Bone removal until you see the whole dural tear

Although this step may seem obvious, in our experience, it is the most common cause of unsuccessful ID repair. As shown in Fig. 4, the dural injury caused by a slipped cutting burr may well exceed the directly offended area and continue underneath the lamina due to the pulling force of the instrument. In addition, in surgery using a tubular retractor the ID may be partially hidden by bone. Therefore, an enlargement of the approach should be considered whenever the border of the dural defect is not completely apparent (Fig. 5).

\section{Intradural look}

This step is especially important in traumatic dural lesions in which the defect may be associated with intradural bone fragments or hematoma-both should be removed. In ID following laceration with a cutting burr, adaptation of transected fibers should be attempted. We used monofile stitches $7 / 0$ or $10 / 0$ or small wrappings with the hemostatic collagen fleece. The end-to-end matching of the right stumps is always a challenge.

3. Repone the fibers

Small cottonoids and low power suction may be helpful to repone $\Omega$-like extruded fibers

4. Do an inside patch if necessary

This step is highly recommended in defects exceeding $5 \mathrm{~mm}$. The hemostatic and sealing surface of the collagen fleece is yellow (riboflavin). Tailor the inside patch roughly $2 \mathrm{~mm}$ larger than the dural gap. The yellow surface should face towards the dura (i.e., outside). The advantages of this inside patching are twofold, as the spinal cord and/or fibers are protected during dural closure. Furthermore, the pulsatile CSF pushes the fleece against the dura, increasing the sealing effect.

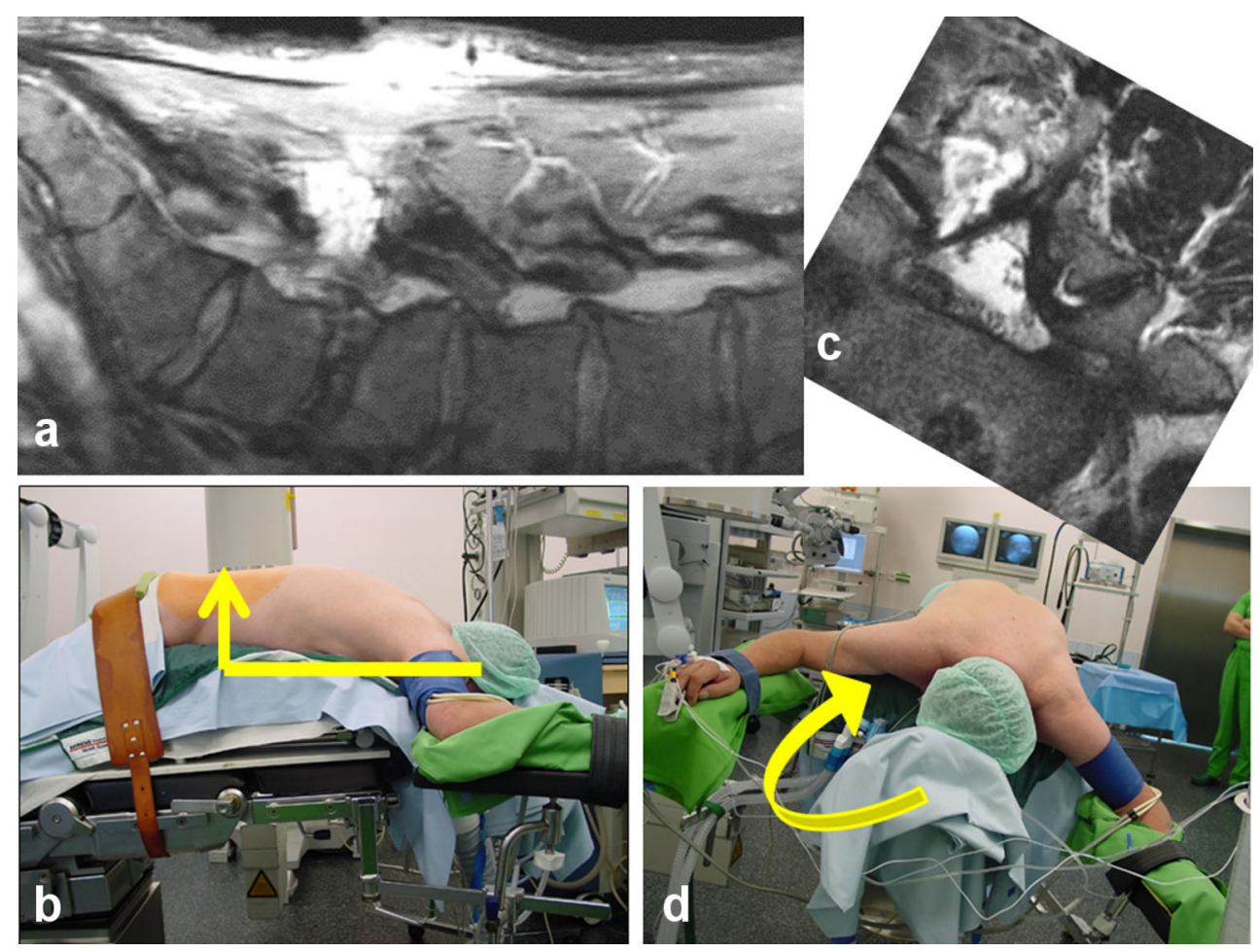

Fig. 2 a T2-weighted sagittal MRI showing a CSF leak at the L4/L5 level. b Positioning on the Wilson frame places the ID $20 \mathrm{~cm}$ higher than the ventricles (yellow arrow). c T2-weighted axial MRI showing the right-sided CSF leak. $\mathbf{d}$ The table is tilted to the left side to elevate the ID to the highest point of the surgical area 


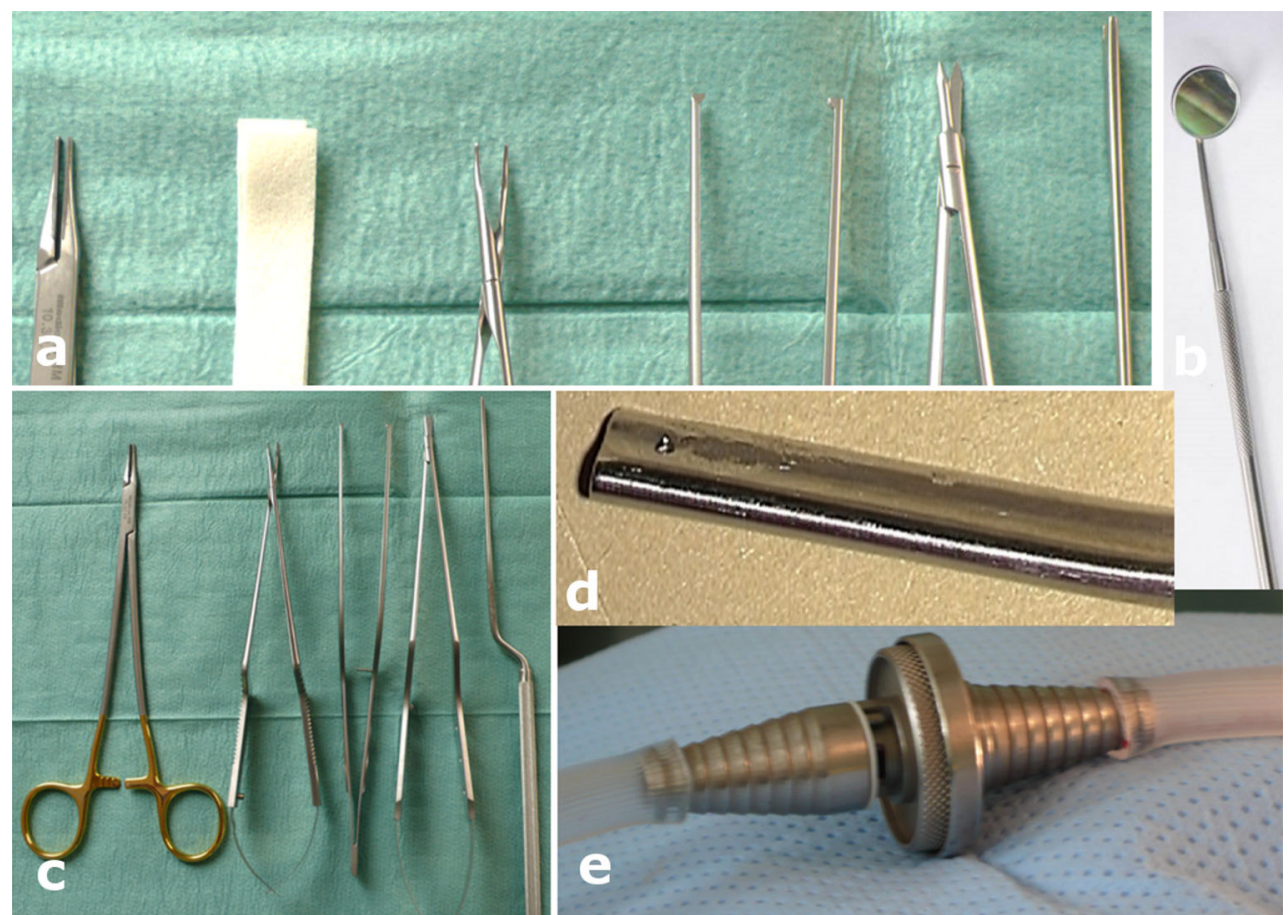

Fig. 3 a Close-up of the tips of the micro-instruments shown in c. b Dental mirror to inspect ventral dural defects. c Bayoneted microinstruments for suturing the dura. d Flat sucker with combined end and front hole to gently push away neurostructures (e.g., caudal fibers). e Valve inserted into the sucker tube to fine tune the sucking power
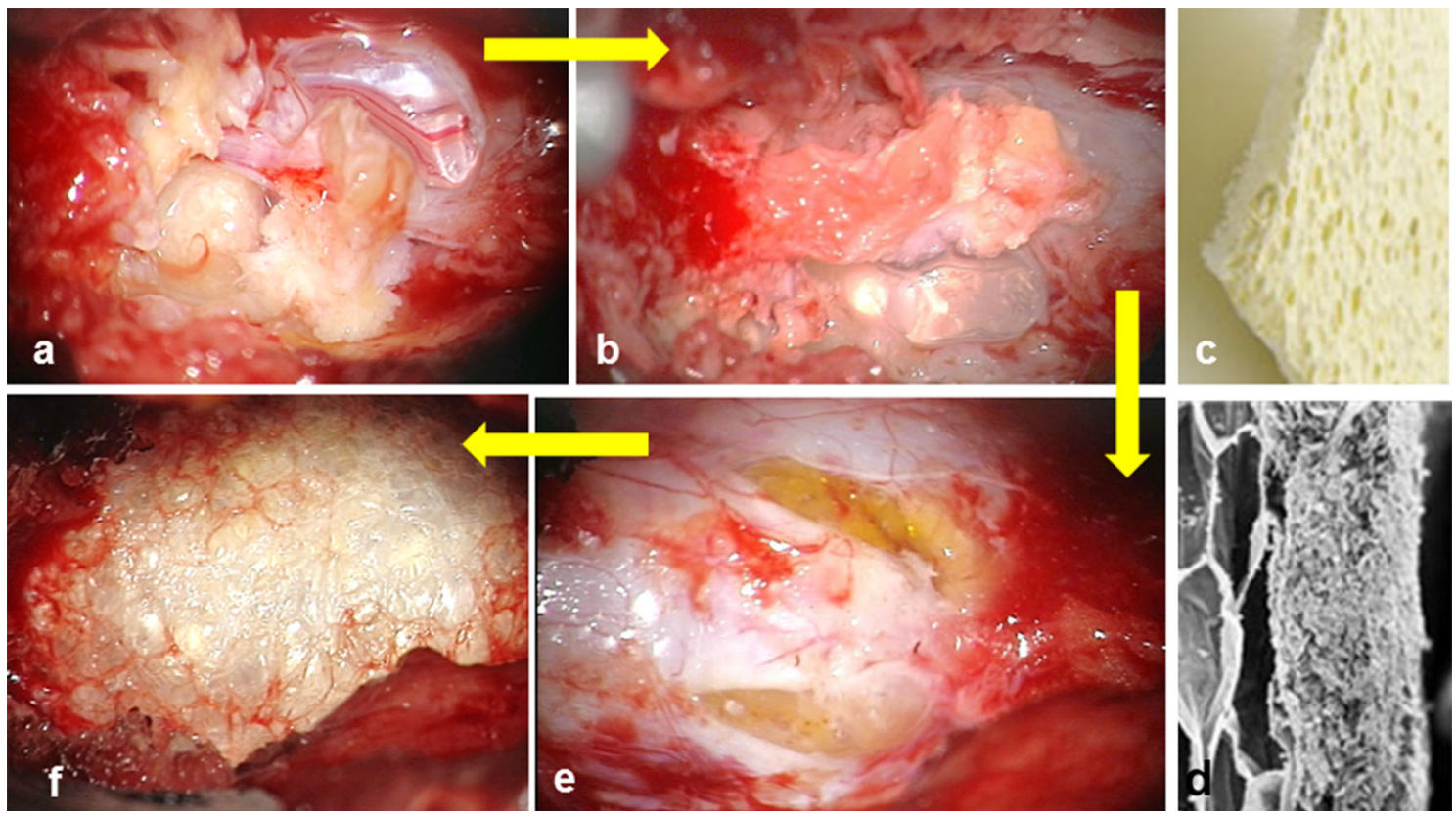

Fig. 4 a Complex ID by cutting burr. Bone, yellow ligament, thecal sac, and caudal fibers are mingled. b A "V"-shaped dural injury appears after partial removal of the bone, always from the periphery towards the dural tear. c Collagen sealant fleece (Tachosil ${ }^{\circledR}$, Takeda $\mathrm{GmbH})$. The yellow surface is the hemostatic/sealant surface. d REM
$(1000 \times)$ showing the transition zone between the trabecular collagen and the sealant layer. e Further bone resection shows the borders of the ID. After repositioning the caudal fibers, an intradural sealant collagen fleece was placed. $f$ The ID was covered by an outside patch of sealant collagen fleece 

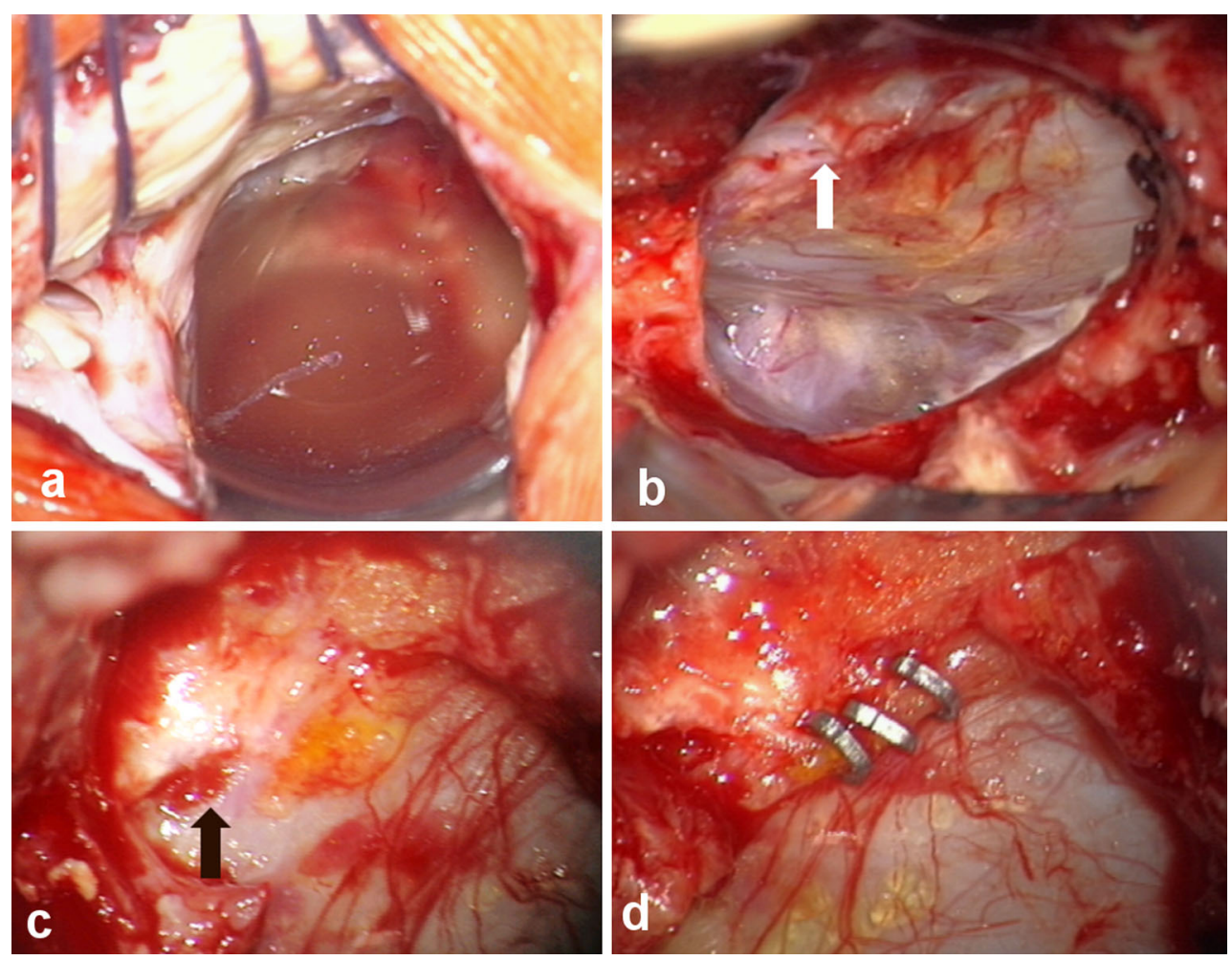

Fig. 5 a A CSF leak became clinically evident 3 days after translaminar removal of a cranially extruded right-sided L4/L5 disc herniation. Intra-operative situs following reopening of the fascia. b A small ID (white arrow) not recognized during the first procedure

\section{Dural closure}

This step has already been described extensively in the literature [5, 12-19]. Primary closure with Prolene 5/0 or $7 / 0$ is our first choice. Whenever access is very limited and hampers the needle holder, an alternative option is nonpenetrating clips, which are usually used in vascular anastomoses, applied by a rotatable shank (AnastoClip ${ }^{\mathrm{TM}} \mathrm{VCS}^{\circledR}$, LeMaitre Inc., USA) [20-22]. If the dural clips cannot be approximated tightly, the defect is covered by a synthetic dural (e.g., DuraGen, Integra Life Sciences Corp., USA) or fascial graft.

\section{Outside patch}

A hemostatic collagen fleece overlapping the borders of the ID is pushed gently with the yellow surface towards the dura. Due to the presence of the inside patch, no pulsatile force will detach the outside patch from the dura (Fig. 4d).The combination of steps 4 and 6 is nicknamed "the sandwich technique". Epidural fibrin glue is also a wellknown option. However, the results are questioned and cases of epidural compression have been reported [13, 14, 23].

\section{Valsalva maneuver}

The positive end-expiratory pressure (PEEP) is raised up to $40 \mathrm{~cm} \mathrm{H}_{2} \mathrm{O}$ and maintained at that level for $30 \mathrm{~s}$. If no CSF leaks out, the repair may be assumed to be effective. became evident. c Close-up view (black arrow). d The ID was closed with three non-penetrating clips grasping a bit of epidural fat due to the small approach via a tubular retractor

This test may be enhanced in equivocal cases by the injection of fluorescein one cranial level from the lesion site.

8. Epidural pedicled muscle flap

The importance of this step cannot be over-emphasized. Although all of the previous steps have failed, the gentle epidural packing (not compression) with muscle tissue prevents CSF from spilling out into a dead space. Even after repeated lumbar punctures, the epidural fat and yellow ligament prevent CSF fistulae because of the lack of an epidural dead space. Tailoring of the muscle flap is shown in Fig. 6. The pedicle of the muscle flap should be anchored into the depth to the spinous process or to the next available bony structure to become uncoupled from the tension of the paravertebral muscles during gait.

9. Multilayer closure

Deep stitches through the bony midline structures further reduce the dead space. A subfascial drain is avoided, though other authors favor it [24]. The superficial layers are closed by back-and-forth sutures and compression dressing enhances their adherence during the first postoperative hours.

10. Drainage of lumbar CSF if necessary

This step is more an exception than a rule. A lumbar CSF drainage may be used if the closure of the ID, mostly 


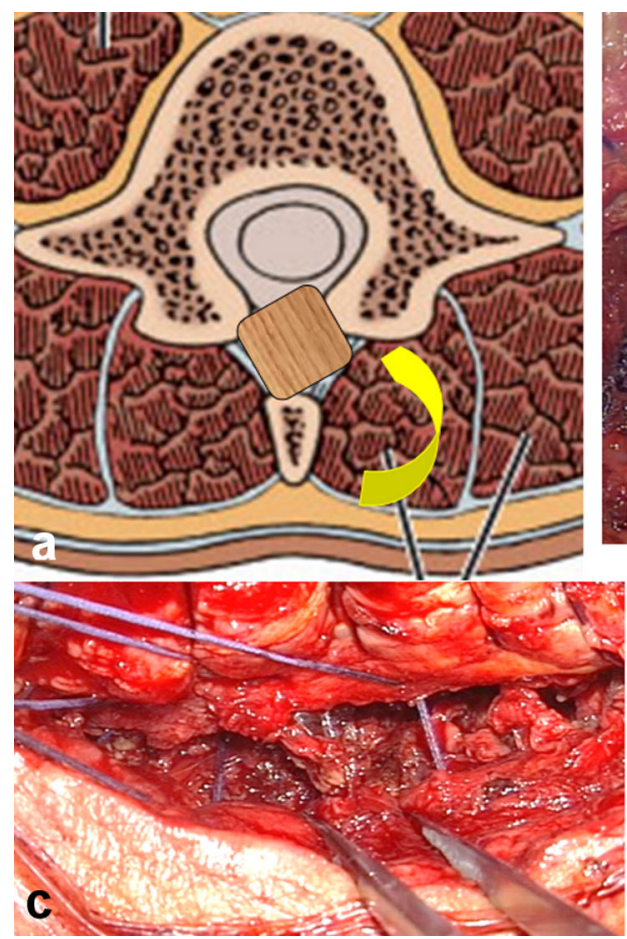

Fig. 6 a Scheme of the pedicled paravertebral muscle flap (brown square) turned down (yellow arrow) to gently pack the epidural space. b Last revision surgery of the "worst case": in situ muscle flap. c Deep stitches anchoring the paravertebral muscles to the spinous

in cases of severe lacerations, is not perfectly tight during the Valsalva maneuver. Lumbar CSF drainage results in CSF hypotension, which may be helpful in revision surgeries for CSF fistulae. We advocate short (3-5 days) but intense (bearable headache, $300-500 \mathrm{ml} /$ daily) drainage.

Postoperative care: ventilation until the next day is indicated whenever tight control of the PEEP is necessary. In this cohort of patients, it was necessary in only three cases, enclosing the "worst case" described later. Bed rest is usually limited to $24 \mathrm{~h}$ but may be prolonged if a lumbar CSF drain is placed. Analgesics are administered generously to prevent abdominal pressure. Stool softeners may also be required.

\section{Results}

The intraspinal part of all 4020 surgeries was performed with the aid of a microscope. The overall prevalence of ID was $4.4 \%$. The prevalence was lowest in virgin microdiscectomies $(1.7 \%$ ), and varied from $3.6 \%$ in decompression for spinal canal stenosis up to $14.5 \%$ in revision procedures. Spinal revision surgery was the most frequent cause $(37 \%)$ of ID repair, followed by decompression of lumbar spinal canal stenosis (32\%), fixation procedures

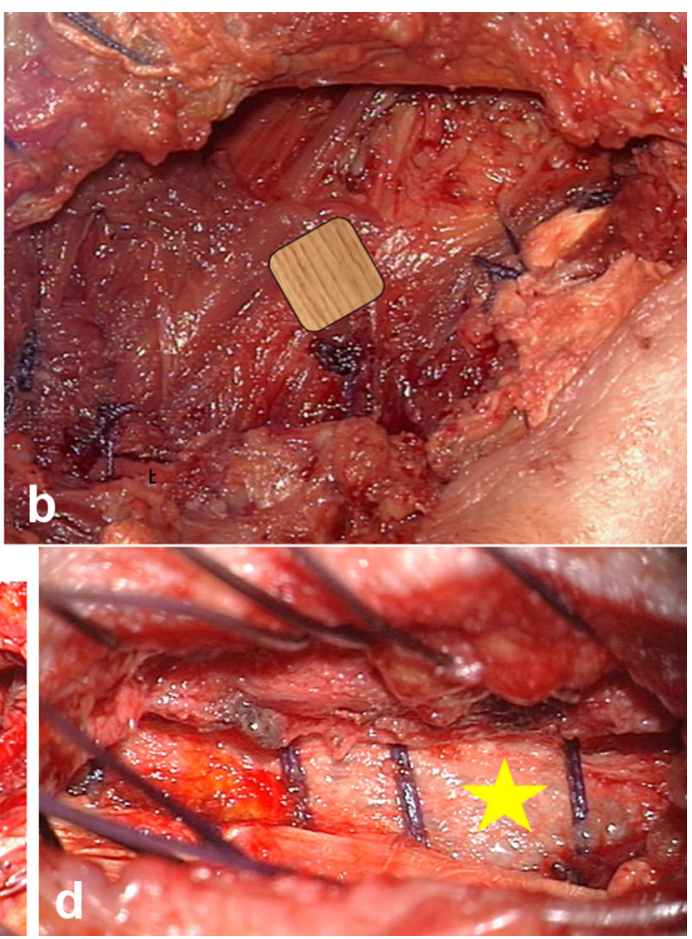

process. d Superficial fascial sutures; a sealant collagen fleece (yellow star) underneath acted as an additional barrier for keeping a potential CSF leak subfascial

(16\%), removal of disc herniation (13\%), and resection of synovial cysts $(2 \%)$.

Among 107 primary surgeries, the InT achieved singlestage closure of the ID in 96 procedures $(89.7 \%)$. Among 20 virgin surgeries, the 10ST was successful in all cases $(P=0.21)$. Among 42 re-do procedures following failed attempts to stop the CSF leakage, the InT achieved singlestage closure in 36 procedures $(85.7 \%)$ and the $10 \mathrm{ST}$ was successful in all 26 cases $(P=0.03)$ (Fig. 7).

The following "worst case" report elucidates how the 10ST may be helpful in difficult dural repair.

A vigorous 92-year-old gentleman underwent microdiscectomy of a large disc herniation at L5/S1 causing excruciating leg pain resistant to CAT-guided infiltrations and 3/5 weakness of the left-sided plantar flexion. The pain disappeared immediately after surgery and the weakness decreased significantly. Two weeks after the initial surgery, a re-do surgery was necessary to remove an interlaminar epidural abscess that had eroded $1 \mathrm{~cm}^{2}$ of the dura. Despite the still intact arachnoid being covered with a sealing collagen fleece, a percutaneous CSF leak developed. Unfortunately, two further revisions could not stop the CSF leak. The patient faced a fifth surgery within 9 weeks. The hope in this dramatic scenario was that antibiotic therapy controlled the infection. 
Fig. 7 The cohort of patients is shown and the efficacy of the InT is compared with the $10 \mathrm{ST}$

195 IDs

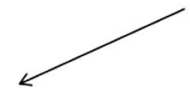

127 virgin surgeries

- 64 Decom for spinal stenosis

- 25 Discectomy

- 20 Monosegmental instrumented decom

- 12 Multisegmental instrumented decom

- 4 Synovial cyst

- 2 Miscellaneous

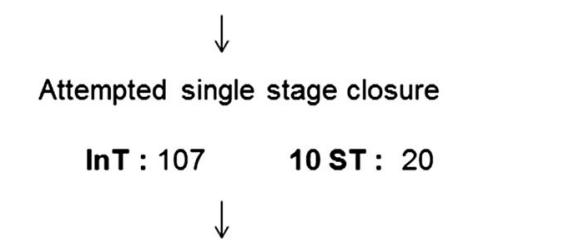

Successful single stage closure

InT : $96(89.7 \%) \quad$ 10ST : $20(100 \%)$

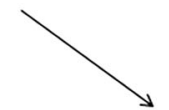

68 revision surgeries

- 55 Own procedures

- 13 External ID referrals

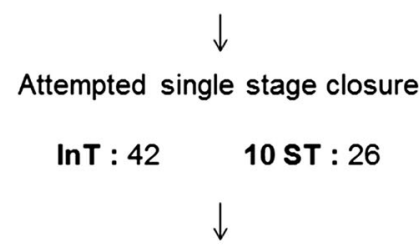

Successful single stage closure

InT : $36(85.7 \%) \quad$ 10ST : $26(100 \%)$

$P=0.03$
The fourth revision procedure started with a resection of the spinous process and L5 lamina. The cleared disc space at L5/S1 was packed with bone chips mixed with a fragmented antibiotic collagen fleece. Fluorescein revealed patency of the axillary dural tear, which was sealed with an intradural and extradural sealant collagen fleece. A large pedicled flap of the right-sided paravertebral muscle was swung onto the epidural area. A tight multilayer closure of the soft tissues was performed (Fig. 6b, d). A CSF drain was inserted at the L4/L5 level. Postoperative ventilation was continued in the ICU over 1 week and the CSF drain was retained for 2 weeks. The subsequent clinical course was complicated by pneumonia and gastric perforation. Both adverse events were treated successfully. The patient was discharged 3 months after the first surgery in reasonably good health.

\section{Discussion}

In the past, different surgeons at our clinic have treated ID in different ways due to the unexpected occurrence and unpredictable severity of ID. In addition, in revision surgery, dural closure, especially of larger defects, was often hampered by extensive scar tissue. Similarly, re-do surgery after failed attempts to stop CSF leakage was challenging because of the previous aggressive action of CSF on tissues. A couple of tools proved to be helpful in improving the quality of ID closure.
The injection of fluorescein has been associated in the literature with complications, such as seizures, and even one death has been reported. However, the single dose of fluorescein that was administered was $100 \mathrm{mg}$ or more in the cases with major complications, and even higher than $500 \mathrm{mg}$ in the mortality case [20]. In a prospective multicenter study, the concentration of the fluorescein solution was lowered to $5 \%$ and the maximum dose limited to $50 \mathrm{mg}$ diluted with $9 \mathrm{ml} \mathrm{CSF}$ and injected slowly. Not even minor adverse effects were reported in 53 patients [21]. The patient has to be informed that the urine will be greenish in color for a couple of days.

The use of collagen fleece with human thrombin and fibrinogen was recommended by the manufacturer to achieve hemostasis. The elasticity of the wet fleece and its sealing side effect were able to withstand pressures of up to $46 \mathrm{mmHg}$ (or $62 \mathrm{~cm} \mathrm{H}_{2} \mathrm{O}$ ), making it very helpful in patching the ID from the intradural and extradural surface. Compared to hydrogeltype sealants, the fleece offers the advantage of an intrinsic structural support, which is especially useful for intrathecal application. The intradural covering of the dural tear protects the spinal cord, as well as the caudal fibers, during the dural closure. Furthermore, the closing function is reinforced by the pulsatile force of the CSF.

The superiority of the 10ST in single-stage repair of complex revision surgery for ID led us to assume that a structured scheme and cadaver lab training before the ID occurs can prepare spinal surgeons, especially trainees, for better handling of the ID. 
One limitation of this report is that the 10ST has not been validated by a prospective or randomized study design. The reason for this is that each ID is very specific, as our "worst case" report showed, and often not comparable with other occurrences. The only common feature is the CSF leak. The second limitation is that the ID cohorts in primary surgery differed markedly in size. Therefore, the comparison of the two treatment options did not reveal any significant difference. Nevertheless, the fact that none of the $4610 \mathrm{ST}$ procedures failed proves the efficacy of the checklist, especially if we consider that single-stage closure was obtained in 26 re-do surgeries, even after multiple unsuccessful attempts to stop the CSF leak.

The strength of this study is that it stresses the need to approach ID with a structured to-do list of surgical steps. We are not aware of this approach being used in a previous publication.

\section{Conclusions}

ID is the most common complication of lumbar spinal surgery. Prevention by carefully planning the sequential surgical steps is of utmost importance. After ID has occurred, intra-operative management tailored to the specific dural tear is the key to prevent a revision procedure for CSF leakage. The 10ST for ID revision results in a significantly higher rate of single-stage dural closure compared to conventional techniques.

\section{Acknowledgments The authors thank Mr. Dieter Witt for sup- porting this study.}

Conflict of interest The authors have no conflict of interest to declare.

Open Access This article is distributed under the terms of the Creative Commons Attribution License which permits any use, distribution, and reproduction in any medium, provided the original author(s) and the source are credited.

\section{References}

1. Leung PC (1988) Complications in the first 40 cases of microdiscectomy. J Spinal Disord 1:306-310

2. Wiese M, Krämer J, Bernsmann K et al (2004) The related outcome and complication rate in primary lumbar microscopic disc surgery depending on the surgeon's experience: comparative studies. Spine 4:550-556

3. McMahon P, Dididze M, Levi AD (2012) Incidental durotomy after spinal surgery: a prospective study in an academic institution. J Neurosurg Spine 17:30-36

4. Takahashi Y, Sato T, Hyodo H et al (2013) Incidental durotomy during lumbar spine surgery: risk factors and anatomic locations. J Neurosurg Spine 18(2):165-169
5. Epstein NE (2007) The frequency and etiology of intraoperative dural tears in 110 predominantly geriatric patients undergoing multilevel laminectomy with noninstrumented fusions. J Spinal Disord Tech 20(5):380-386

6. Baker GA, Cizik AM, Bransford RJ et al (2012) Risk factors for unintended durotomy during spine surgery: a multivariate analysis. Spine J 12(2):121-126

7. Guerin P, El Fegoun AB, Obeid I et al (2012) Incidental durotomy during spine surgery: incidence, management and complications. A retrospective review. Injury 43(4):397-401

8. Kalevski SK, Peev NA, Haritonov DG (2010) Incidental dural tears in lumbar decompressive surgery: incidence, causes, treatment, results. Asian J Neurosurg 5(1):54-59

9. Desai A, Ball PA, Bekelis K et al (2012) Surgery for lumbar degenerative spondylolisthesis in Spine Patient Outcomes Research Trial: does incidental durotomy affect outcome? Spine 37(5):406-413

10. Desai A, Ball PA, Bekelis K et al (2011) SPORT: does incidental durotomy affect long-term outcomes in cases of spinal stenosis? Neurosurgery 69(1):38-44

11. Jones AA, Stambough JL, Balderston RA et al (1989) Long-term results of lumbar spine surgery complicated by unintended incidental durotomy. Spine 14(4):443-446

12. Ruban D, O'Toole JE (2011) Management of incidental durotomy in minimally invasive spine surgery. Neurosurg Focus 31(4):E15

13. Jankowitz BT, Atteberry DS, Gerszten PC et al (2009) Effect of fibrin glue on the prevention of persistent cerebral spinal fluid leakage after incidental durotomy during lumbar spinal surgery. Eur Spine J 18(8):1169-1174

14. Mulder M, Crosier J, Dunn R (2009) Cauda equina compression by hydrogel dural sealant after a laminotomy and discectomy: case report. Spine 34(4):E144-E148

15. Cammisa FP, Girardi FP, Sangani PK et al (2000) Incidental durotomy in spine surgery. Spine 25(20):2263-2267

16. Bosacco SJ, Gardner MJ, Guille JT (2001) Evaluation and treatment of dural tears in lumbar spine surgery: a review. Clin Orthop Relat Res 389:238-247

17. Khan MH, Rhin J, Steele G et al (2006) Postoperative management protocol for incidental dural tears during surgery: a review of 3183 consecutive degenerative lumbar cases. Spine 31(22): 2609-2613

18. Eismont FJ, Wiesel SW, Rothmann RH (1981) Treatment of dural tears associated with spinal surgery. J Bone Jt Surg (Am) 63:1132-1136

19. Dafford EE, Anderson PA (2013) Comparison of dural repair techniques. Spine J. doi:10.1016/j.spinee.2013.06.44 (in press)

20. Keerl R, Weber RK, Draf W et al (2004) Use of sodium fluorescein solution for detection of cerebrospinal fluid fistulas: an analysis of 420 administrations and reported complications in Europe and the United States. Laryngoscope 114:266-272

21. Felisati G, Bianchi A, Lozza P et al (2008) Italian multicentre study on intrathecal fluorescein for craniosinusal fistulae. Acta Otorhinolaryngol Ital 28(4):159-163

22. Park P, Leveque JC, La Marca F et al (2010) Dural closure using the U-clip in minimally invasive spinal tumor resection. J Spinal Disord Tech 23:486-489

23. Epstein NE (2010) Dural repair with four spinal sealants: focused review of the manufacturers' inserts and the current literature. Spine J 10(12):1065-1068

24. Wang JC, Bohlmann HH, Riew DK (1998) Dural tears secondary to operations on the lumbar spine: management and results after a two-year-minimum follow-up of eighty-eight patients. J Bone Jt Surg (Am) 80:1728-1732 